\title{
EICHLER, Daniel, Fränkische Reichsversammlungen unter Ludwig dem Frommen
}

\section{Gaëlle Calvet-Marcade}

\section{OpenEdition}

Journals

Édition électronique

URL : http://journals.openedition.org/ifha/1800

DOI : $10.4000 /$ ifha. 1800

ISSN : 2198-8943

Éditeur

IFRA - Institut franco-allemand (sciences historiques et sociales)

Référence électronique

Gaëlle Calvet-Marcade, «EICHLER, Daniel, Fränkische Reichsversammlungen unter Ludwig dem

Frommen », Revue de l'IFHA [En ligne], Date de recension, mis en ligne le 01 janvier 2009, consulté le 22 septembre 2020. URL : http://journals.openedition.org/ifha/1800 ; DOI : https://doi.org/10.4000/ifha. 1800

Ce document a été généré automatiquement le 22 septembre 2020.

(C)IFHA 


\title{
EICHLER, Daniel, Fränkische Reichsversammlungen unter Ludwig dem Frommen
}

\author{
Gaëlle Calvet-Marcade
}

Cette étude fait partie des travaux préparatoires à l'édition des actes de Louis le Pieux (814-840) dans la célèbre collection des MGH et a pour objectif de donner une nouvelle définition des assemblées générales d'Empire (Reichsversammlungen). Il est aujourd'hui évident que ces assemblées sont au cœur des pratiques du pouvoir carolingien et représentent la réalité du gouvernement à cette époque. Cette étude se trouve dans le courant actuel de la médiévistique qui, depuis quelques temps, remet en question les modèles explicatifs hérités de l'école juridique du XIXe s. Loin de la vision figée de l'histoire institutionnelle, l'assemblée d'Empire devient un temps de rencontre qu'il faut redéfinir à chaque fois.

D.E. commence par questionner les mots des sources et le vocabulaire des historiens où le même flou terminologique existe. Il étudie les substantifs désignant ces assemblées : conventus, placitum, concilium, synodus, mais aussi les pronoms possessifs (suus et noster) et les adjectifs (publicus, generale, sanctus) qui les accompagnent. La variété des termes latins pouvant désigner une assemblée a grandement influencé les historiens qui ont cherché à établir une classification cohérente des différents types d'assemblée. D.E. présente et déconstruit chacune de ces catégories : plaid, synode, consilium regis, assemblée militaire, diète (Reichstag) sont l'expression d'une même réalité politique, une assemblée générale d'empire tenue en présence de l'empereur.

Pour lui, la notion de Reichsversammlung n'est pas un concept cadre englobant tous les types d'assemblée, ni le terme générique pour des assemblées de grande importance par rapport à des conseils réduits. Une assemblée générale d'Empire désigne toute assemblée tenue en présence de l'empereur et où peuvent être prises des décisions politiques sur les affaires du royaume. Tous les autres critères, comme le nombre et la qualité des participants, la nature des sujets traités (religieux, civil, militaire) ou la portée de cette assemblée, ne jouent aucun rôle dans la définition. 
Après avoir ainsi délimité le concept, D.E. poursuit son étude par l'analyse des lieux et des temps de rencontre. Les 61 plaids du règne de Louis le Pieux sont suffisamment bien datés et localisés pour lui permettre une recherche assez fine. La répartition chronologique et le choix du lieu dépendent autant des conditions matérielles que des événements politiques. L'auteur poursuit son analyse par l'étude du cercle des participants renvoyant notamment aux travaux de P. Depreux, qui a identifié 275 personnes ayant participé à un plaid sous le règne de Louis le Pieux.

Puis il s'intéresse au déroulement et aux modalités de fonctionnement de l'assemblée. Il étudie les différents moyens utilisés par l'empereur pour convoquer les grands, mais aussi la durée et le contenu de l'assemblée. Trois fonctions principales se dégagent : l'œuvre législative, la justice, puis le gouvernement et l'administration du royaume. C'est là que se manifeste le plus la rencontre entre l'empereur et les grands, par la discussion des affaires à traiter, les pratiques de négociation et la recherche du consensus. C'est aussi un moyen pour l'empereur de garder un lien avec les grands éloignés de lui et de s'informer de la situation du royaume. En cela, l'assemblée générale est le lieu d'information et d'échange entre l'empereur et les grands, mais aussi des grands entre eux.

D.E. résume en deux mots les assemblées du règne de Louis le Pieux : flexibilité et ouverture. Ces temps de rencontre sont d'une grande plasticité, ils permettent d'aborder toutes les affaires importantes et de prendre des décisions. Les assemblées répondent à un souci pragmatique d'efficacité, ce sont des instruments concrets du pouvoir impérial mais aussi des hauts lieux de la représentation symbolique des rapports de force.

On trouve à la fin de l'ouvrage des cartes avec les différents lieux de rencontre, des diagrammes sur la répartition chronologique et la fréquence, ainsi qu'un tableau récapitulatif des assemblées.

Gaëlle CALVET-MARCADE (Université Paris I Panthéon-Sorbonne) 